\title{
Over-Expressing Prohibitin (PHB) in Neuronal Cultures Exacerbates Cell Death Following Hydrogen Peroxide and L-Glutamic Acid Induced Injury
}

\author{
Jonathan Teoh', Sherif Boulos ${ }^{1}$, Joanne Chieng1, Neville W. Knuckey ${ }^{1,2}$, Bruno P. Meloni ${ }^{1,2^{*}}$ \\ ${ }^{1}$ Centre for Neuromuscular and Neurological Disorders, The University of Western Australia and Western \\ Australian Neuroscience Research Institute, QEII Medical Centre, Nedlands, WA, Australia \\ ${ }^{2}$ Department of Neurosurgery, Sir Charles Gairdner Hospital, Nedlands, WA, Australia \\ Email: "bruno.meloni@anri.uwa.edu.au
}

Received 3 June 2014; revised 2 July 2014; accepted 1 August 2014

Copyright @ 2014 by authors and Scientific Research Publishing Inc.

This work is licensed under the Creative Commons Attribution International License (CC BY). http://creativecommons.org/licenses/by/4.0/

(c) () Open Access

\begin{abstract}
Using proteomics, previous work in our laboratory identified five mitochondrial related proteins [citrate synthase (CS), glucose-regulated protein 75 (GRP75), heat shock protein 60 (HSP60), prohibitin (PHB), voltage-dependent anion channel 1 (VDAC1)] to be differentially expressed in primary cortical neuronal cultures following preconditioning treatments [1] [2]. To investigate a protective or damaging role of these five proteins in neurons, we used RNAi constructs to knockdown and adenoviral vectors to over-express the proteins in cortical neuronal cultures prior to exposure to three ischemia-related injury models: excitotoxicity (L-glutamic acid), oxidative stress (hydrogen peroxide) and in vitro ischemia (oxygen-glucose deprivation). We observed that downregulating these mitochondrial proteins had no effect on neuronal viability, in any injury model. By contrast, over-expression of PHB exacerbated cell death in the hydrogen peroxide and L-glutamic acid injury models. These findings indicate that PHB plays a neurodamaging role following oxidative and excitotoxic stress and suggests that the protein is a potential therapeutic target for the design of drugs to limit neuronal death following cerebral ischemia and other forms of brain injury.
\end{abstract}

\section{Keywords}

Prohibitin, Neurodamage, Mitochondria, In Vitro Injury Models, Neuronal Cultures

\footnotetext{
"Corresponding author.
}

How to cite this paper: Teoh, J., Boulos, S., Chieng, J., Knuckey, N.W. and Meloni, B.P. (2014) Over-Expressing Prohibitin (PHB) in Neuronal Cultures Exacerbates Cell Death Following Hydrogen Peroxide and L-Glutamic Acid Induced Injury. Neuroscience \& Medicine, 5, 149-160. http://dx.doi.org/10.4236/nm.2014.54018 


\section{Introduction}

Acute brain injury following stroke and other forms of cerebral ischemia have an immerse impact not only on patients and their families but also on the broader community, due to the high social and economic cost. Major risk factors for stroke include hypertension, smoking, lack of physical activity, diabetes and an unhealthy diet [3]. Brain damage following a stroke manifests itself through a range of symptoms including problems with coordination and speech, paralysis in one or more limbs, dizziness, visual defects and reduced alertness or concentration [4]. Presently, as neuroprotective agents capable of limiting ischemic brain injury are unavailable, a major objective in stroke research is the identification of potential therapeutic targets for the development of clinically effective neuroprotective drugs. One approach to identify therapeutic targets for neuroprotective drug discovery is to investigate differential protein expression following neuronal preconditioning or the induction of ischemic tolerance. Preconditioning occurs when mild non-damaging stimuli or exogenous factors activate endogenous adaptive mechanisms that can protect neurons against a subsequent damaging insult. As preconditioning is reliant on new protein synthesis, the identification of proteins involved in this process is a potential target in the development of drugs to inhibit ischemic neuronal death.

In earlier studies conducted in our laboratory [1] [2] we utilised four (heat stress: $42.5^{\circ} \mathrm{C}$ for $1 \mathrm{~h}$; erythropoietin: $12 \mathrm{~h}$ exposure; cycloheximide: exposure for $24 \mathrm{~h}$; MK801: 30 min exposure) treatments that induce preconditioning in cultured primary neuronal cultures. Using proteomics we subsequently identified five mitochondria-related proteins that were either up- or down-regulated following preconditioning. These proteins included citrate synthase (CS), glucose-regulated protein 75 (GRP75), heat shock protein 60 (HSP60), prohibitin (PHB) and voltage-dependent anion channel 1 (VDAC1).

As cerebral ischemia can affect mitochondria both positively and negatively to alter cell viability, it is likely that proteins associated with mitochondrial function represent therapeutic targets. For example, during cerebral ischemia mitochondria initially attempt to conserve ATP, sequester intracellular calcium, and release reactive oxygen species (ROS) that may stimulate cell survival pathways [5]. In contrast, ischemic stress can cause mitochondrial disturbances including impairments to ATP generation, calcium overload, excessive ROS production and the release of proteins that promote cell death [6]. Therefore, based on the importance of mitochondria in controlling neuronal fate following ischemia we decided to characterize the neuroprotective or neurodamaging role of five mitochondrial-related proteins identified in our earlier proteomic studies [1] [2]. To do this, we investigated how knock-down or over-expression of each protein impacted on the viability of primary cortical neuronal cultures in three different ischemia-related injuries models (i.e. hydrogen peroxide and L-glutamic acid, oxygen-glucose deprivation).

\section{Materials and Methods}

\subsection{Primary Neuronal Cortical Cultures}

All animal procedures were approved by the University of Western Australia Animal Ethics Committee. Establishment of cortical cultures was as previously described [7]. Briefly, cortical tissue from E18-E19 SpragueDawley rats (sourced from Animal Care Services, The University of Western Australia) was dissociated in Dulbecco's modified Eagle medium (DMEM; Invitrogen, Australia) supplemented with $1.3 \mathrm{mM}$ L-cysteine, 0.9 $\mathrm{mM} \mathrm{NaHCO}_{3}, 10$ units/ml papain (Sigma, USA) and 50 units/ml DNase (Sigma) and washed in cold DMEM/10\% horse serum. Neurons were resuspended in Neurobasal (Invitrogen) containing 2\% B27 supplement (B27; Invitrogen). Before seeding, 96-well sized glass wells (7 mm diameter, Grace Davison Discovery Sciences, Australia) or 96-well plastic plates (Nunc, Australia) were coated with poly-D-lysine overnight ( $50 \mu \mathrm{l} / \mathrm{well}$ : $50 \mathrm{mg} / \mathrm{ml}$; 70 - 150 K, Sigma) followed by incubation with $60 \mu \mathrm{l}$ of Neurobasal (containing 2\% B27; 4\% fetal bovine serum; 1\% horse serum; $62.5 \mu \mathrm{M}$ L-glutamic acid; $25 \mu \mathrm{M}$ 2-mercaptoethanol; and $30 \mu \mathrm{g} / \mathrm{ml}$ streptomycin and 30 $\mu \mathrm{g} / \mathrm{ml}$ penicillin) for $1-2$ hours at $37^{\circ} \mathrm{C}$ in $\mathrm{CO}_{2}$ incubator ( $5 \% \mathrm{CO}_{2} / 95 \%$ air). Culture wells were plated with neurons in $90 \mu$ of Neurobasal/2\% B27 to obtain $\approx 10,000$ viable neurons for each well on day in vitro 11 - 12 . Neuronal cultures were maintained in $\mathrm{CO}_{2}$ incubator at $37^{\circ} \mathrm{C}$. On day in vitro 4, one third of the culture medium was removed and replaced with fresh Neurobasal/2\% B27 containing the mitotic inhibitor, cytosine arabinofuranoside ( $1 \mu \mathrm{M}$ final concentration; Sigma). On day in vitro 8 , one half of the culture medium was replaced with Neurobasal/2\% B27. Cultures were used on day in vitro 11 or 12 after which time they consist of $>97 \%$ neurons and $1 \%-3 \%$ astrocytes [7]. 


\subsection{Short Interfering RNA (siRNA) Treatment of Neuronal Cultures}

On day in vitro 6, media was removed from the cortical neuronal cultures and replaced with $50 \mu \mathrm{l}$ of Neurobasal/2\% (v/v) B27 media containing $500 \mathrm{nM}$ of a Dharmacon Accell siRNA constructs (siRNA:CS, siRNA:GRP75, siRNA:HSP60, siRNA:PHB, and siRNA:VDAC1; Thermo Fisher Scientific, USA) and incubated at in a $\mathrm{CO}_{2}$ incubator at $37^{\circ} \mathrm{C}$ for 48 hours (note: no transfection reagent was used). On day in vitro 8, an equal volume of $\mathrm{NB} / 2 \% \mathrm{~B} 27$ media containing $500 \mathrm{nM}$ siRNA construct was added to the wells and cultures incubated for a further 72 hours before being used for protein isolation or being subjected to injury models. The Accell siRNA non-targeting RNAi construct (siRNA:NT) was used as a control in all experiments.

\subsection{Generation and Amplification of Adenoviral Vector and Adenoviral Treatment of Neuronal Cultures}

Recombinant adenoviral vectors were prepared using the AdEasy vector system (Qbiogene, [8]), with some modifications as described previously [9]. The full-length gene of interest (GOI) cDNA for rat CS, GRP75, HSP60, PHB, and VDAC1 sequences were subcloned into our modified shuttle plasmid vector designated pRSV/WPRE; CMV:EGFP [9] to create the plasmid pRSV:GOI/WPRE; CMV:EGFP (pRSV:GOI). Subsequently, pRSV:GOI DNA was linearized by PmeI digestion and introduced by electroporation (Gene Pulser II, Biorad) into Escherichia coli strain BJ5183 carrying pAdEasy [10]. A recombinant (i.e. a bacterial colony derived from a bacterium that had undergone homologous recombination with the pRSV:GOI and pAdEasy plasmids) was selected on media containing $50 \mu \mathrm{g} / \mathrm{ml}$ kanamycin, and plasmid DNA checked by PacI digestion (i.e. homologous recombination). Lipofectamine 2000 (Invitrogen) was used to transfect PacI enzyme linearized pRSV:GOI/AdEasy recombinant DNA (3 $\mu$ g) into HEK293 cells (QBiogene) grown in a $25 \mathrm{~cm}^{2}$ flask. Following the appearance of viral plaques (5 days), viral amplification was performed in a step-wise fashion by infecting HEK293 cells in $175 \mathrm{~cm}^{2}$ and $500 \mathrm{~cm}^{2}$ flasks. HEK293 cell lysate-containing adenoviral particles were prepared by three rounds of freezing/thawing before concentration using the Adeno-X virus purification kit (BD Biosciences). The viral titre was determined by the end-point dilution assay as indicated by EGFP reporter expression [11]. The GOI expressing adenoviral vectors, which also express the EGFP reporter under the control of a CMV vector were designated: AdRSV:CS, AdRSV:GRP75, AdRSV:HSP60, AdRSV:PHB and AdRSV:VDAC1. An empty vector (AdRSV:Empty) that also express the EGFP reporter have been described previously [9] and were used as controls.

On day in vitro 9, the media was removed from cortical neuronal cultures and replaced with Neurobasal/2\% B27 media containing purified adenovirus at a multiplicity of infection (moi) of 75 . Cultures were incubated for a further 48 hours before total protein was isolated for western blot analysis or used for injury models.

\subsection{SDS PAGE and Western Blotting}

Total protein was isolated from neuronal cultures by treatment with lysisbuffer [50 mM Tris- $\mathrm{HCl} \mathrm{pH} 7.5,100$ mM NaCl, 20 mM EDTA, 0.1\% (v/v) SDS, 0.2\% (w/v) deoxycholic acid, 0.25\% (v/v) Ipegal (Sigma), Complete $^{\mathrm{TM}}$ protease inhibitor, (Roche)] followed by centrifugation at 20,000 rpm for 5 minutes at $4^{\circ} \mathrm{C}$. Protein concentration in samples was determined using the Bradford protein assay. Protein samples (10 $\mu$ g/sample) were loaded onto $10 \%(\mathrm{v} / \mathrm{v})$ or $4 \%$ - $12 \%$ SDS poly-acrylamide Bis-Tris mini gels (NuPAGE, Invitrogen) and separated via electrophoresis for 50 minutes at a constant voltage (200 volts). Separated proteins were electrotransferred at 30 volts for 1 hour onto polyvinylidinedifluoride or nitrocellulose membranes (Pall Corp., USA). Membranes were washed with 0.1\% (v/v) Tween 20 in PBS (PBS/T) for 15 minutes before being blocked with 5\% (v/v) skim milk in PBS/T for 1 hour at room temperature. Membranes were incubated with primary antibody (rabbit anti-CS, Sigma; rabbit anti-GRP75, Santa Cruz; Lab Vision/Neo Markers; mouse anti-HSP60, Lab Vision/Neo Markers; rabbit anti-PHB, Santa Cruz; goat anti-VDAC1; BD Biosciences) in PBS/T containing ovalbumin $(1 \mathrm{mg} / \mathrm{ml})$ at $4^{\circ} \mathrm{C}$ overnight. After washing with PBS/T for 15 minutes, the membrane was incubated with a horseradish peroxidase conjugated secondary antibody (donkey anti-rabbit, GE Healthcare; sheep anti-mouse, GE Healthcare; rabbit anti-goat, Zymed) in PBS/T containing ovalbumin for 1 hour at room temperature. Immuno-reactive bands were visualized using an enhanced chemiluminescence detection system (Amersham, UK) and exposure to X-ray film (Hyperfilm, Amersham). For immuno-detection of the $\beta$-tubulin loading control protein, membranes were incubated for 10 minutes at room temperature with stripping solution (Alpha Diagnos- 
tic, USA) prior to re-probing with a mouse anti- $\beta$-tubulin anti-body (BD Biosciences).

\subsection{Neuronal in Vitro Injury Models}

\subsubsection{In Vitro Ischemia Model (Oxygen-Glucose Deprivation)}

In order to expose rat cortical neuronal cultures to in vitro ischemia (oxygen-glucose deprivation), media was removed from each well and washed with $315 \mu \mathrm{l}$ of BSS (pH 6.9), followed by the re-addition of $60 \mu \mathrm{l}$ of BSS. Culture wells were placed in an anaerobic chamber (Don Whitely Scientific, England) with an atmosphere of 5\% $\mathrm{CO}_{2}, 10 \% \mathrm{H}_{2}$ and $85 \%$ argon at $98 \%$ humidity and $37^{\circ} \mathrm{C}$ for 40 or 45 minutes ( 40 min was used for adenoviral vector experiments as it was observed that neurons were slightly more sensitive to injury). Following removal from the anaerobic incubator, an equal volume of Neurobasal media containing 2\% N2 supplement (Invitrogen) was added into each well, and cultures placed in a $\mathrm{CO}_{2}$ incubator at $37^{\circ} \mathrm{C}$. Control cultures were subjected to the same wash procedures only, and maintained in a $\mathrm{CO}_{2}$ incubator. Neuronal viability was assessed at 24 hour after the initiation of in vitro ischemia.

\subsubsection{Hydrogen Peroxide Model (Oxidative Stress)}

Media from neuronal culture wells was removed and replaced with $100 \mu \mathrm{l}$ of Neurobasal/1\% N2 supplement containing 3 or $3.5 \mu \mathrm{M}$ of hydrogen peroxide ( $3 \mu \mathrm{M}$ was used for adenoviral vector experiments). Cultures were incubated at $37^{\circ} \mathrm{C}$ in an incubator (5\% $\mathrm{CO}_{2} / 95 \%$ air) for 1 hour, after which the medium from each well was removed and replaced with $100 \mu \mathrm{l}$ of Neurobasal $/ 1 \% \mathrm{~N} 2$ supplement. Cultures were incubated at $37^{\circ} \mathrm{C}$ in a $\mathrm{CO}_{2}$ incubator for a further 24 hours. Controls cultures received the same media changes but were not exposed to hydrogen peroxide. Neuronal viability was assessed at 24 hours after hydrogen peroxide exposure.

\subsubsection{L-Glutamic Acid Model (Excitotoxicity)}

Media in neuronal culture wells was removed and replaced with $50 \mu \mathrm{l}$ of Neurobasal/2\% B27 supplement media containing L-glutamic acid (6 or $25 \mu \mathrm{M}$ was used for adenoviral vector experiments). Cultures were incubated at $37^{\circ} \mathrm{C}$ in a $\mathrm{CO}_{2}$ incubator for 5 minutes, after which time the medium from each well was removed and replaced with $100 \mu \mathrm{l}$ of fresh Neurobasal/1\% N2 supplement before being incubated at $37^{\circ} \mathrm{C}$ in a $\mathrm{CO}_{2}$ incubator for a further 24 hours. Control cultures were treated the same but without exposure to L-glutamic acid containing media. A positive control consisted of cultures containing the glutamate receptor antagonists, MK801 (5 $\mu \mathrm{M})$ and 6-cyano-7-nitroquinoxaline (5 $\mu \mathrm{M}$; CNQX) during L-glutamic acid exposure. Neuronal viability was assessed at 24 hours after L-glutamic acid exposure.

\subsection{Neuronal Viability Assay and Statistical Analysis}

Neuronal cell cultures were examined by light microscopy for qualitative assessment of neuronal cell viability 18 - 24 hours after in vitro injury, and quantitatively after 24 hours by the colorimetric 3-(4,5-dimethyliazol-2-yl)5-(3-carboxymethoxyphenyl)-2-(4-sulfophenyl)-2H-tetrazoliumsalt (MTS) assay. MTS absorbance data was converted to reflect proportional cell viability relative to both the untreated (taken as $100 \%$ viable) and treated controls. For all models, three independent experiments were performed with quadruplicate (L-glutamic acid and hydrogen peroxide exposure) or sextuplicate (oxygen-glucose deprivation) replicate samples. Data obtained was pooled for generating graphs and for analysis by ANOVA followed by post-hoc Fischer's PLSD test, with $P$ $<0.05$ values being considered as statistically significant.

\section{Results}

\subsection{Down-Regulation of Proteins and Effect on Neuronal Viability in Injury Models}

Western blot analysis confirmed that siRNA constructs for CS, GRP75, HSP60, PHB and VDAC1decreased the expression of their respective target protein in cortical neuronal cultures (Figure 1). Cortical neuronal cultures treated with specific siRNA constructs and subjected to in vitro ischemia, hydrogen peroxide and L-glutamic acid exposure, did not result in any statistically significant increase or decrease in neuronal viability (Figure 2). However there was a slight trend of increased neuronal survival in cultures treated with the siRNA:CS and siRNA:PHB constructs following hydrogen peroxide exposure $(P$ values: siRNA:CS $=0.43$, siRNA:PHB $=$ $0.25)$. L-glutamic acid receptor blockers significantly increased neuronal viability following L-glutamic acid exposure $(P$ value $=0.0023$; Figure $2(C)$ ). 


\section{A}

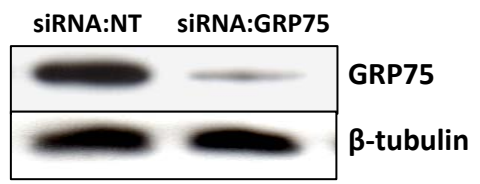

C

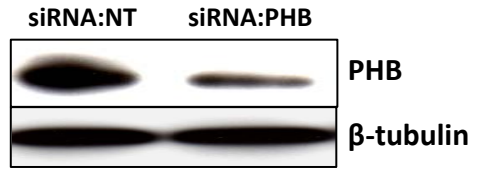

B

SIRNA:NT SIRNA:HSP60

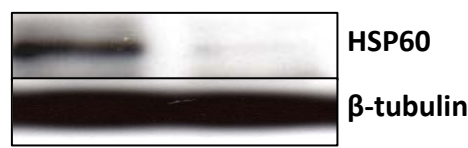

D

SIRNA:NT SIRNA:VDAC1

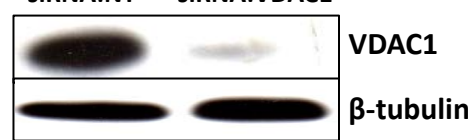

E

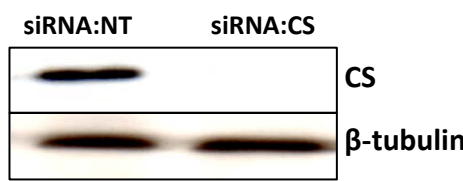

Figure 1. Western blot analysis of cortical neuronal cultures treated with RNAi constructs (500 $\mathrm{nM}$ ) and probed with specific protein antibodies. To ensure similar protein loading in control (siRNA non-targeting; NT) and treated samples membranes were probed with an anti- $\beta$-tubulin antibody. (A) siRNA:NT and siRNA:GRP75 and probed with anti-GRP75 antibody; (B) siRNA:NT and siRNA:HSP60 and probed with anti-HSP60 antibody; (C) siRNA:NT and siRNA:PHB and probed with anti-PHB antibody; (D) siRNA:NT and siRNA: VDAC1 and probed with anti-VDAC1 antibody; (E) siRNA:NT and siRNA: CS and probed with anti-CS antibody.

A

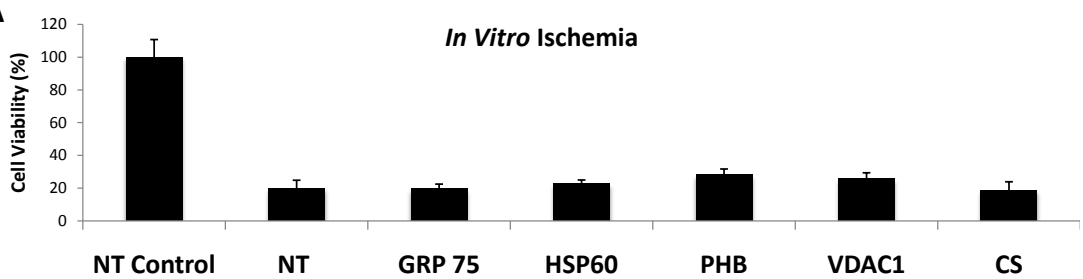

B

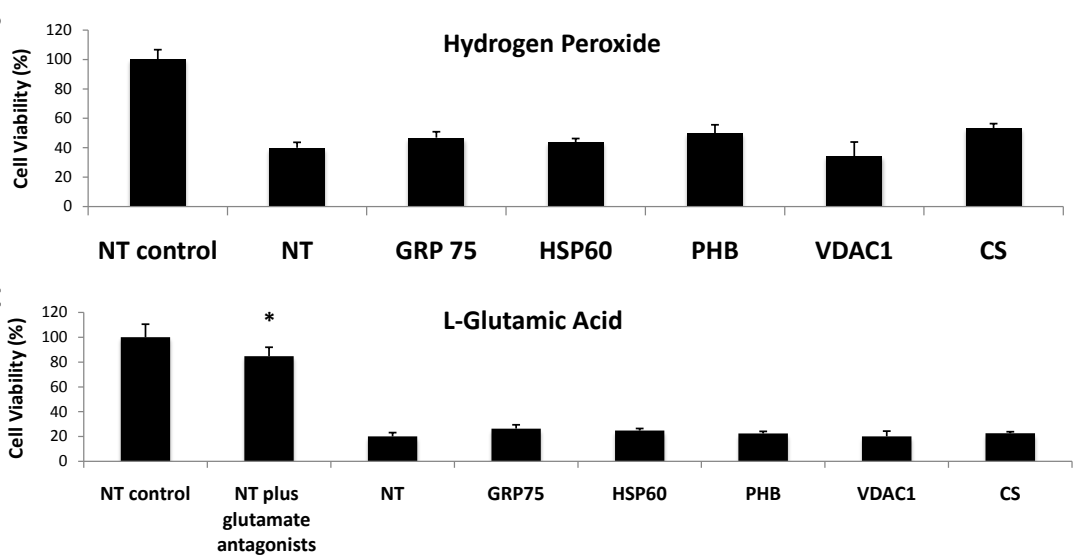

Figure 2. Cell viability of neuronal cultures treated with siRNA constructs (NT = siRNA:NT, GRP75 = siRNA:GRP75, HSP60 = siRNA:HSP60, PHB = siRNA:PHB, VDAC1 = siRNA:VDAC1 and CS = siRNA:CS; $500 \mathrm{Nm}$ ) at 24 hours following. (A) In vitro ischemia (45 min, $\mathrm{n}=18$ ); (B) Hydrogen peroxide exposure (3.5 $\mu \mathrm{M}, \mathrm{n}=12)$; (C) L-glutamic acid exposure $(25 \mu \mathrm{M}, \mathrm{n}=12)$. The glutamate receptor antagonists used were $5 \mu \mathrm{M}$ MK801/5 $\mu \mathrm{M}$ CNQX. Control cultures (NT control) treated with siRNA: NT were taken as $100 \%$ cell survival. Values are expressed as means $\pm \operatorname{SD}\left({ }^{*} P<0.05\right)$. 


\subsection{Up-Regulation of Proteins and Effect on Neuronal Viability in Injury Models}

Western blot analysis confirmed that adenoviral vectors for CS, GRP75, HSP60, PHB and VDAC1 increased the expression of their respective protein in cortical neuronal cultures (Figure 3). Results obtained for the three injury models are described below.

\subsubsection{In Vitro Ischemia Model}

Cortical neuronal cultures transduced withadenoviral vectors expressing specific proteinsand subjected to in vitro ischemia had no effect on neuronal viability compared to control adenoviral vector treated cultures (Figure 4).

A

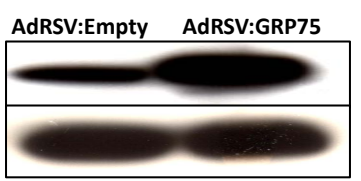

C

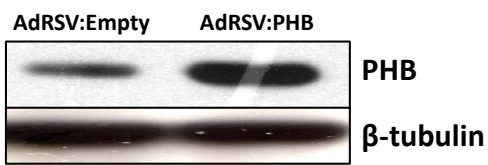

B

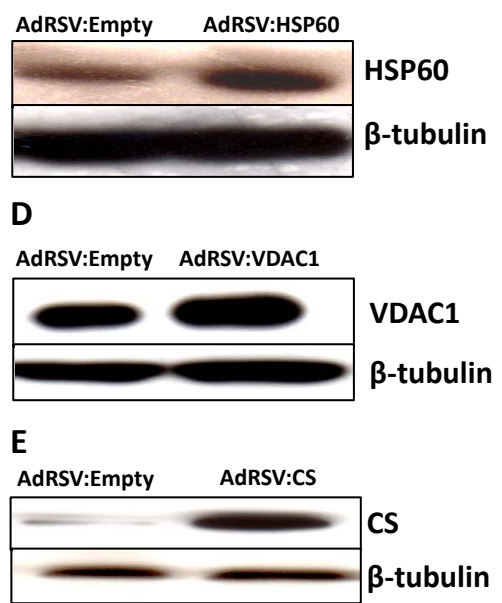

Figure 3. Western blot analysis of cortical neuronal cultures transfected with adenoviral vectors (at a moi of 100) and probed with specific protein antibodies and anti- $\beta$-tubulin antibody (loading control protein). (A) AdRSV:Empty and AdRSV:GRP75 and probed with anti-GRP75 antibody; (B) AdRSV:Empty and AdRSV:HSP60 and probed with anti-HSP60 antibody; (C) AdRSV:Empty and AdRSV:PHB and probed with anti-PHB antibody; (D) AdRSV:Empty and AdRSV:VDAC1 and probed with anti-VDAC1 antibody; (E) AdRSV: Empty and AdRSV:CS and probed with anti-CS antibody.

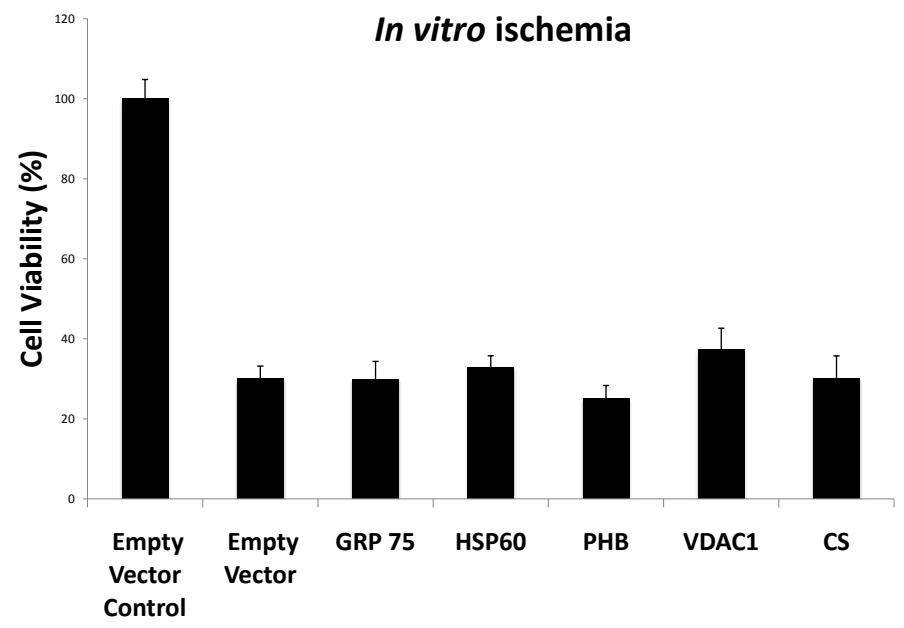

Figure 4. Cell viability of neuronal cultures treated with adenoviral vectors (AdRSV: Empty, AdRSV:GRP75, AdRSV:HSP60, AdRSV:PHB, AdRSV:VDAC1 and AdRSV: $\mathrm{CS}$; moi of 75$)$ at 24 hours following in vitro ischemia (40 min). Control neuronal cultures (empty vector control) treated with AdRSV: Empty and not exposed to in vitro ischemia were taken as $100 \%$ cell survival. Values are expressed as means \pm SD ( $n=18$; $P<0.05)$. 


\subsubsection{Hydrogen Peroxide Model}

Cortical neuronal cultures transduced with the AdRSV:PHB adenoviral vector and exposed to hydrogen peroxide decreased neuronal viability compared to control adenoviral vector treated cultures (Figure 5, $P$ value $=$ 0.03). Over-expression of the other proteins (CS, GRP75, HSP60, and VDAC1) had no effect on cell viability.

\subsubsection{L-Glutamic Acid Model}

Cortical neuronal cultures transduced with the AdRSV:PHB adenoviral vector and exposed to L-glutamic acid decreased neuronal viability compared to control adenoviral vector treated cultures (Figure 6, $P$ value $=0.003$ ). Over-expression of the other proteins (CS, GRP75, HSP60, and VDAC1) had no effect on cell viability. Treatment of cultures with the empty vector plus glutamate receptor blockers also significantly increased neuronal viability in this model.

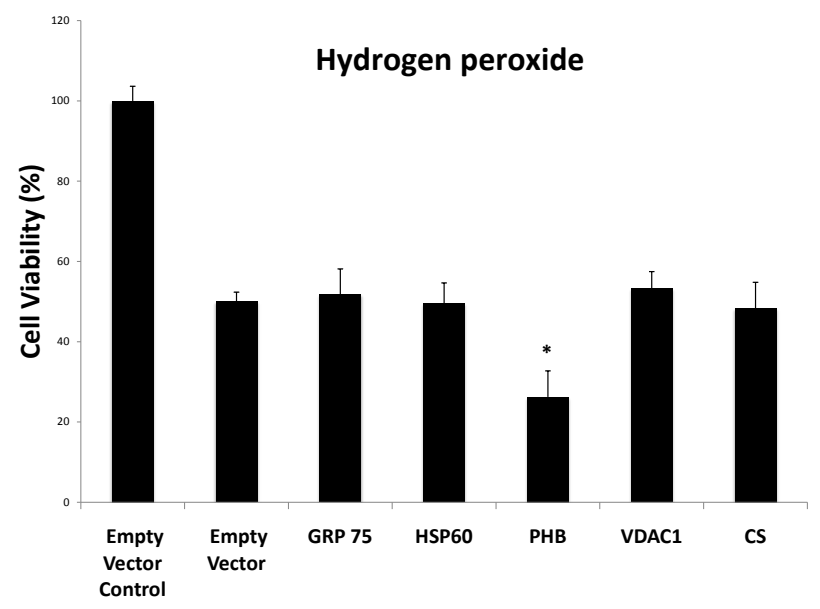

Figure 5. Cell viability of neuronal cultures treated with adenoviral vectors (AdRSV:Empty, AdRSV:GRP75, AdRSV:HSP60, AdRSV:PHB, AdRSV: VDAC1 and AdRSV:CS; moi of 75) at 24 hours following hydrogen peroxide exposure $(3 \mu \mathrm{M})$. Control neuronal cultures (empty vector control) treated with AdRSV:Empty and not exposed to hydrogen peroxide were taken as $100 \%$ cell survival. Values are expressed as means \pm SD $\left(n=12 ;{ }^{*} P<0.05\right)$.

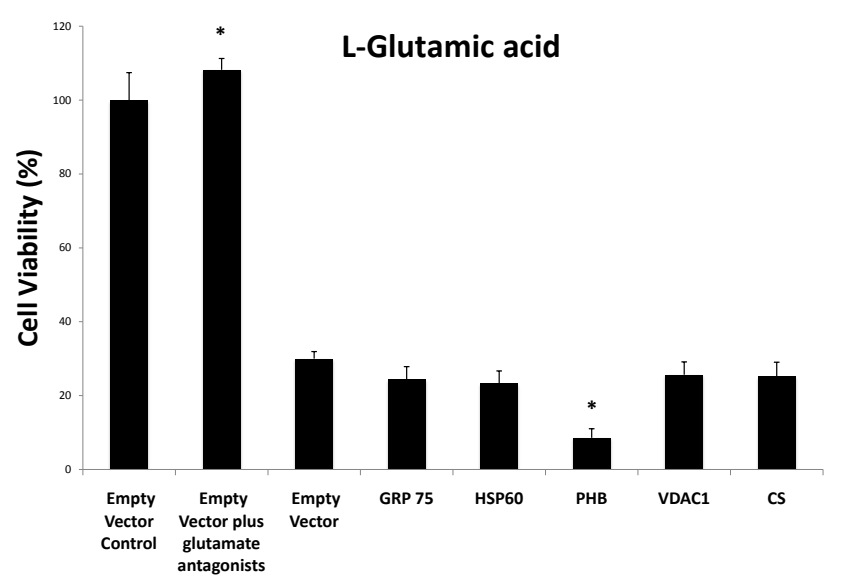

Figure 6. Cell viability of neuronal cultures treated with adenoviral vectors (AdRSV:Empty, AdRSV:GRP75, AdRSV:HSP60, AdRSV:PHB, AdRSV: VDAC1 and AdRSV:CS; moi of 75) at 24 hours following L-glutamic acid exposure $(6 \mu \mathrm{M})$. The glutamate receptor antagonists used were $5 \mu \mathrm{M}$ MK $801 / 5 \mu \mathrm{M}$ CNQX. Control neuronal cultures (empty vector control) treated with AdRSV:Empty and not exposed to L-glutamate were taken as $100 \%$ cell survival. Values are expressed as means $\pm \mathrm{SD}\left(n=12 ;{ }^{*} P<0.05\right)$. 


\section{Discussion}

In this study, three in vitro injury models (in vitro ischemia, L-glutamic acid excitotoxicity and hydrogen peroxide oxidative stress) were used to assess the effects of down-regulation and up-regulation of five mitochondrialrelated proteins on neuronal viability. The down-regulation of the proteins did not induce any significant decrease or increase in neuronal death. This is in contrast to previous studies reporting that down-regulating PHB exacerbated neuronal cell death following oxidative stress and L-glutamic acid exposure [12]. In addition, treat ment of rat brain slices with an anti-VDAC1 antibody reduces neuronal death following in vitro ischemia and L-glutamic acid excitotoxicity [13]. These discrepancies could be related to differences in the cell cultures and injury models used, and the degree of protein down-regulation. Moreover, given that protein down-regulation did not cause any significant negative effects in cell viability following injury, it is possible that the reduction in a specific protein may have activated adaptive signalling or metabolic pathways.

The key finding in this study was the demonstration that PHB protein up-regulation exacerbated neuronal cell death following hydrogen peroxide and L-glutamic acid injury. By contrast, the up-regulation of the other four proteins (CS, GRP75, HSP60, VDAC1) examined did not have any significant effect on neuronal outcome in the three injury models.

Prohibitin (PHB) is a member of the Band-7 family of proteins. Band-7 proteins are associated with a range membrane-associated processes such as, modulating the activity membrane-bound proteases, assembly of mitochondria respiratory complexes, ion channel function and vesicle trafficking. While PHB is mainly localised in the mitochondria, it is also found in the nucleus and plasma membrane [14] [15]. In mitochondria, PHB interacts with the protein TFAM (mitochondria transcription factor A) to maintain mitochondrial DNA stability. TFAM is part of the mitochondrial nucleoprotein complex (nucleoids) that regulates the stability, packaging, replication, transcription and maintenance of mitochondrial DNA [16]. PHB also interacts with another mitochondrial protein, OPA1 (optic atrophy protein 1 homolog), which is a dynamin-like GTPase located within the mitochondrial inter-membrane space that is essential for mitochondrial fusion and cristae morphogenesis [17]. In contrast to our findings, recent in vitro and in vivo studies have reported a protective role for PHB over-expression following ischemic insults [12]. Given its diverse functions, it is not surprising that altering cellular PHB levels can have both a negative and positive impact on cell survival.

Over-expression of PHB in cardiomyocytes helps maintain mitochondrial membrane potential leading to improved cell survival during hypoxia [18] and is protective against exogenous hydrogen peroxide exposure [19]. The exact mechanism whereby PHB protects against hypoxic and oxidative stress is not fully understood, but maybe related to its ability to regulate the electron transport chain enzyme, cytochrome coxidase [20]. For example, RNA interference knockdown of PHB in epithelial cells causes an increase in mitochondrial ROS production due to a decrease of complex I activity and blockade of the electron transport chain [21]. This mechanism may also in part contribute to the damaging effects of PHB down-regulation in neuronal cells following oxidative stress and L-glutamic acid exposure. However, while PHB may have positive effects on cells, the protein can also participate in cell damaging pathways. For example, it has been shown that PHB up-regulation increases the expression of the pro-apoptotic protein Bax, activates the stress-activated c-Jun N-terminal kinases (JNK) pathway and promotes the activation of caspase-3, caspase-9, p53 and poly (ADP-ribose) polymerase (PARP) [22] [23]. Hence, it is clear that PHB over-expression has the potential to exacerbate neuronal injury through the activation of apoptosis.

While the current study showed that up-regulation of the other four proteins had no significant effects of on neuronal viability following ischemia-like insults, this is in contrast with some previous published studies. A study has demonstrated that over-expression of GRP75 was neuroprotective against in vitro ischemia [24]. Moreover, it was reported that plasmid DNA mediated over-expression of GRP75 in both as trocytes and neurons decreases infarct volume in model of focal cerebral ischemia [25]. Similarly, adenoviral mediated overexpression of HSP60 is neuroprotective in a model of global ischemia [26]. The contrasting findings of our results compared to other studies could be related to differences in the injury models used (e.g. insult severity, neuronal density and maturation, in vitro versus in vivo systems, non-neuronal cell populations), over-expression procedures and degree and duration of protein up-regulation. Further experiments, possibly using transgenic mice over-expressing specific proteins could provide more definitive data to determine if the proteins investigated in the present study have a positive or negative role on neurons in the is chaemic brain. 


\section{Conclusion}

In conclusion, the down-regulation of mitochondrial-related proteins had no effect on neuronal viability following three in vitro injury models (in vitro ischemia, L-glutamic acid excitotoxicity and hydrogen peroxide oxidative stress). However, our finding that PHB up-regulation has a pronounced neurodamaging effect following oxidative stress and excitotoxicity suggests that it or its downstream pathways may be a potential target for the design of drugs to limit neuronal death following cerebral ischemia and brain trauma.

\section{Acknowledgements}

This study was supported by the Neuromuscular Foundation of Western Australia, the Sir Charles Gairdner Hospital Research Fund, and by a University of Western Australia postgraduate research scholarship to Jonathan Teoh.

\section{Conflict of Interest}

The authors have no competing financial interests.

\section{References}

[1] Meloni, B.P., Tilbrook, P.A., Boulos, S., Arthur, P.G. and Knuckey, N.W. (2006) Erythropoietin Preconditioning in Neuronal Cultures: Signaling, Protection from in Vitro Ischemia, and Proteomic Analysis. Journal of Neuroscience Research, 83, 584-593. http://dx.doi.org/10.1002/jnr.20755

[2] Meloni, B.P., Van Dyk, D., Cole, R. and Knuckey, N.W. (2005) Proteome Analysis of Cortical Neuronal Cultures Following Cycloheximide, Heat Stress and MK801 Preconditioning. Proteomics, 5, 4743-4753. http://dx.doi.org/10.1002/pmic.200500107

[3] O’Donnell, M.J., et al. (2010) Risk Factors for Ischaemic and Intracerebral Haemorrhagic Stroke in 22 Countries (the INTERSTROKE Study): A Case-Control Study. Lancet, 376, 112-123. http://dx.doi.org/10.1016/S0140-6736(10)60834-3

[4] Schretzman, D. (2001) Acute Ischemic Stroke. Dimensions of Critical Care Nursing, 20, 14-21. http://dx.doi.org/10.1097/00003465-200103000-00005

[5] Maragos, W.F. and Korde, A.S. (2004) Mitochondrial Uncopling as a Potential Therapeutic Target in Acute Central Nervous System Injury. Journal of Neurochemistry, 91, 257-262.

[6] Galluzzi, L., Morselli, E., Kepp, O. and Kroemer, G. (2009) Targeting Post-Mitochondrial Effectors of Apoptosis for Neuroprotection. Biochimico et Biophysica Acta, 1787, 402-413.

[7] Meloni, B.P., Majda, B.T. and Knuckey, N.W. (2001) Establishment of Neuronal in Vitro Models of Ischemia in 96-Well Microtiter Strip-Plates That Result in Acute, Progressive and Delayed Neuronal Death. Neuroscience, 108, 1726. http://dx.doi.org/10.1016/S0306-4522(01)00396-7

[8] He, T.C., et al. (1998) A Simplified System for Generating Recombinant Adenoviruses. Proceedings of the National Academy of Sciences USA, 95, 2509-2514. http://dx.doi.org/10.1073/pnas.95.5.2509

[9] Boulos, S., Meloni, B.P., Arthur, P.G., Bojarski, C. and Knuckey, N.W. (2006) Assessment of CMV, RSV and SYN1 Promoters and the Woodchuck Post-Transcriptional Regulatory Element in Adenovirus Vectors for Transgene Expression in Cortical Neuronal Cultures. Brain Research, 1102, 27-38. http://dx.doi.org/10.1016/j.brainres.2006.04.089

[10] Zeng, M., et al. (2001) AdEasy System Made Easier by Selecting the Viral Backbone Plasmid Preceding Homologous Recombination. Biotechniques, 31, 260-262.

[11] Luo, J., et al. (2007) A Protocol for Rapid Generation of Recombinant Adenoviruses Using the AdEasy System. Nature Protocols, 2, 1236-1247. http://dx.doi.org/10.1038/nprot.2007.135

[12] Zhou, P., et al. (2012) Prohibitin Reduces Mitochondrial Free Radical Production and Protects Brain Cells from Different Injury Modalities. Journal of Neuroscience, 32, 583-592. http://dx.doi.org/10.1523/JNEUROSCI.2849-11.2012

[13] Perez Velazquez, J.L., Kokarovtseva, L., Weisspapir, M. and Frantseva, M.V. (2003) Anti-Porin Antibodies Prevent Excitotoxic and Ischemic Damage to Brain Tissue. Journal of Neurotrauma, 20, 633-647. http://dx.doi.org/10.1089/089771503322144554

[14] Fusaro, G., Dasgupta, P., Rastogi, S., Joshi, B. and Chellappan, S. (2003) Prohibitin Induces the Transcriptional Activity of p53 and Is Exported from the Nucleus upon Apoptotic Signaling. The Journal of Biological Chemistry, 278, 47853-47861. http://dx.doi.org/10.1074/jbc.M305171200

[15] Terashima, M., et al. (1994) The IgM Antigen Receptor of B Lymphocytes Is Associated with Prohibitin and a Pro- 
hibitin-Related Protein. The EMBO Journal, 13, 3782-3792.

[16] Chen, X.J. and Butow, R.A. (2005) The Organization and Inheritance of the Mitochondrial Genome. Nature Reviews Genetics, 6, 815-825. http://dx.doi.org/10.1038/nrg1708

[17] Hoppins, S., Lackner, L. and Nunnari, J. (2007) The Machines That Divide and Fuse Mitochondria. Annual Review of Biochemistry, 76, 751-780. http://dx.doi.org/10.1146/annurev.biochem.76.071905.090048

[18] Muraguchi, T., Kawawa, A. and Kubota, S. (2010) Prohibitin Protects against Hypoxia-Induced H9c2 Cardiomyocyte Cell Death. Biomedical Research, 31, 113-122. http://dx.doi.org/10.2220/biomedres.31.113

[19] Liu, X., et al. (2009) Prohibitin Protects against Oxidative Stress-Induced Cell Injury in Cultured Neonatal Cardiomyocyte. Cell Stress and Chaperones, 14, 311-319. http://dx.doi.org/10.1007/s12192-008-0086-5

[20] Tsutsumi, T., et al. (2009) Proteomics Analysis of Mitochondrial Proteins Reveals Overexpression of a Mitochondrial Protein Chaperon, Prohibitin, in Cells Expressing Hepatitis C Virus Core Protein. Hepatology, 50, 378-386. http://dx.doi.org/10.1002/hep.22998

[21] Schleicher, M., et al. (2008) Prohibitin-1 Maintains the Angiogenic Capacity of Endothelial Cells by Regulating Mitochondrial Function and Senescence. Journal of Cell Biology, 180, 101-112. http://dx.doi.org/10.1083/jcb.200706072

[22] Liu, Y.H., Peck, K. and Lin, J.Y. (2012) Involvement of Prohibitin Upregulation in Abrin-Triggered Apoptosis. Evidence-Based Complementary and Alternative Medicine, 2012, Article ID: 605154. http://dx.doi.org/10.1155/2012/605154

[23] Zhang, L., Ji, Q., Ni, Z.H. and Sun, J. (2012) Prohibitin Induces Apoptosis in BGC823 Gastric Cancer Cells through the Mitochondrial Pathway. Asian Pacific Journal of Cancer Prevention, 13, 3803-3807. http://dx.doi.org/10.7314/APJCP.2012.13.8.3803

[24] Voloboueva, L.A., et al. (2008) Overexpression of Mitochondrial Hsp70/Hsp75 Protects Astrocytes against Ischemic Injury in Vitro. Journal of Cerebral Blood Flow \& Metabolism, 28, 1009-1016. http://dx.doi.org/10.1038/sj.jcbfm.9600600

[25] Xu, L., Voloboueva, L.A., Ouyang, Y., Emery, J.F. and Giffard, R.G. (2009) Overexpression of Mitochondrial Hsp70/ Hsp75 in Rat Brain Protects Mitochondria, Reduces Oxidative Stress, and Protects from Focal Ischemia. Journal of Cerebral Blood Flow \& Metabolism, 29, 365-374. http://dx.doi.org/10.1038/jcbfm.2008.125

[26] Hwang, I.K., et al. (2007) Changes in Immunoreactivity of HSP60 and Its Neuroprotective Effects in the Gerbil Hippocampal CA1 Region Induced by Transient Ischemia. Experimental Neurology, 208, 247-256. http://dx.doi.org/10.1016/j.expneurol.2007.08.017 


\section{List of Abbreviations}

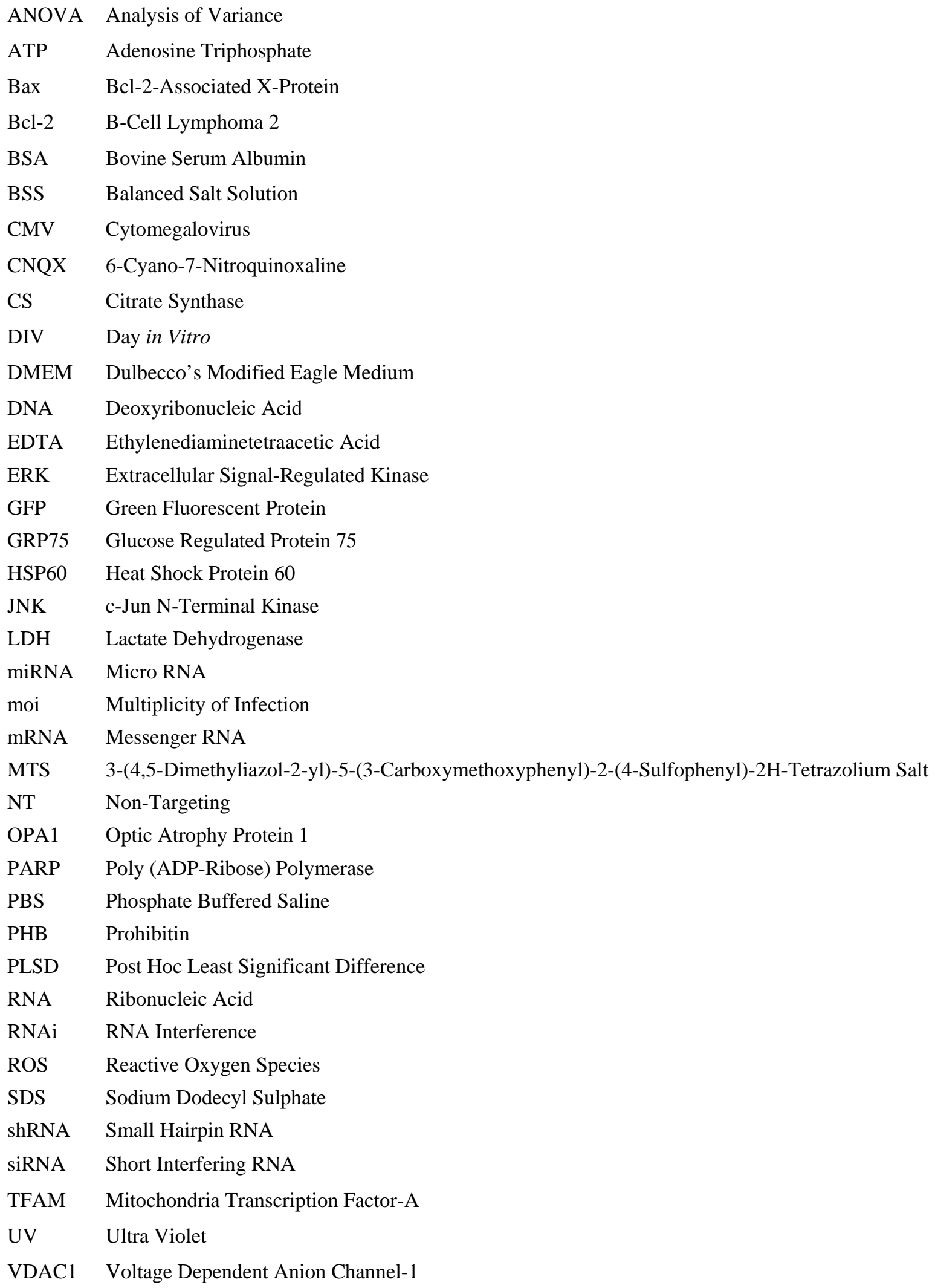


J. Teoh et al.

$\begin{array}{ll}\text { OD } & \text { Optical Density } \\ \mathrm{L} & \text { Litre } \\ \mathrm{ml} & \text { Millilitre } \\ \mu \mathrm{l} & \text { Microlitre } \\ \mathrm{g} & \text { Gram } \\ \mathrm{mg} & \text { Milligram } \\ \mu \mathrm{g} & \text { Microgram } \\ \mathrm{ng} & \text { Nanogram } \\ \mathrm{kDa} & \text { Kilo Daltons } \\ \mathrm{M} & \text { Molar } \\ \mathrm{mM} & \text { Millimolar } \\ \mu \mathrm{M} & \text { Micromolar } \\ \mathrm{nM} & \text { Nanomolar } \\ { }^{\circ} \mathrm{C} & \text { Degrees Celcius } \\ \mathrm{nm} & \text { Nanometer }\end{array}$


Scientific Research Publishing (SCIRP) is one of the largest Open Access journal publishers. It is currently publishing more than 200 open access, online, peer-reviewed journals covering a wide range of academic disciplines. SCIRP serves the worldwide academic communities and contributes to the progress and application of science with its publication.

Other selected journals from SCIRP are listed as below. Submit your manuscript to us via either submit@scirp.org or Online Submission Portal.
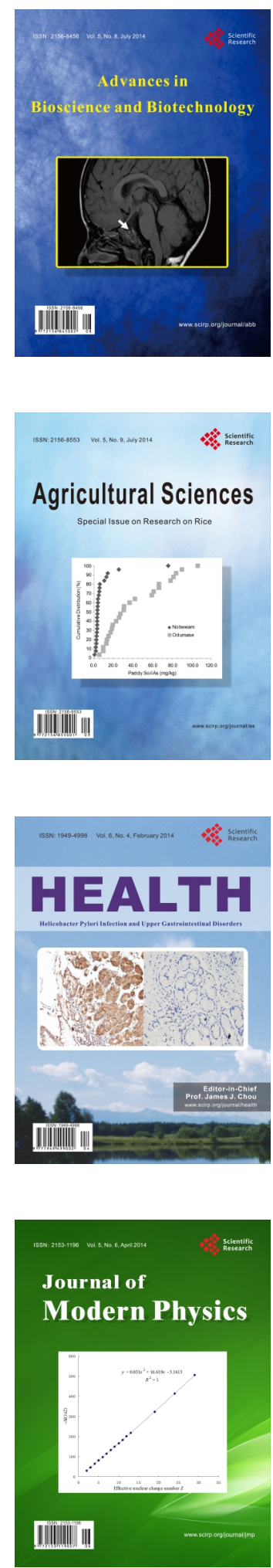
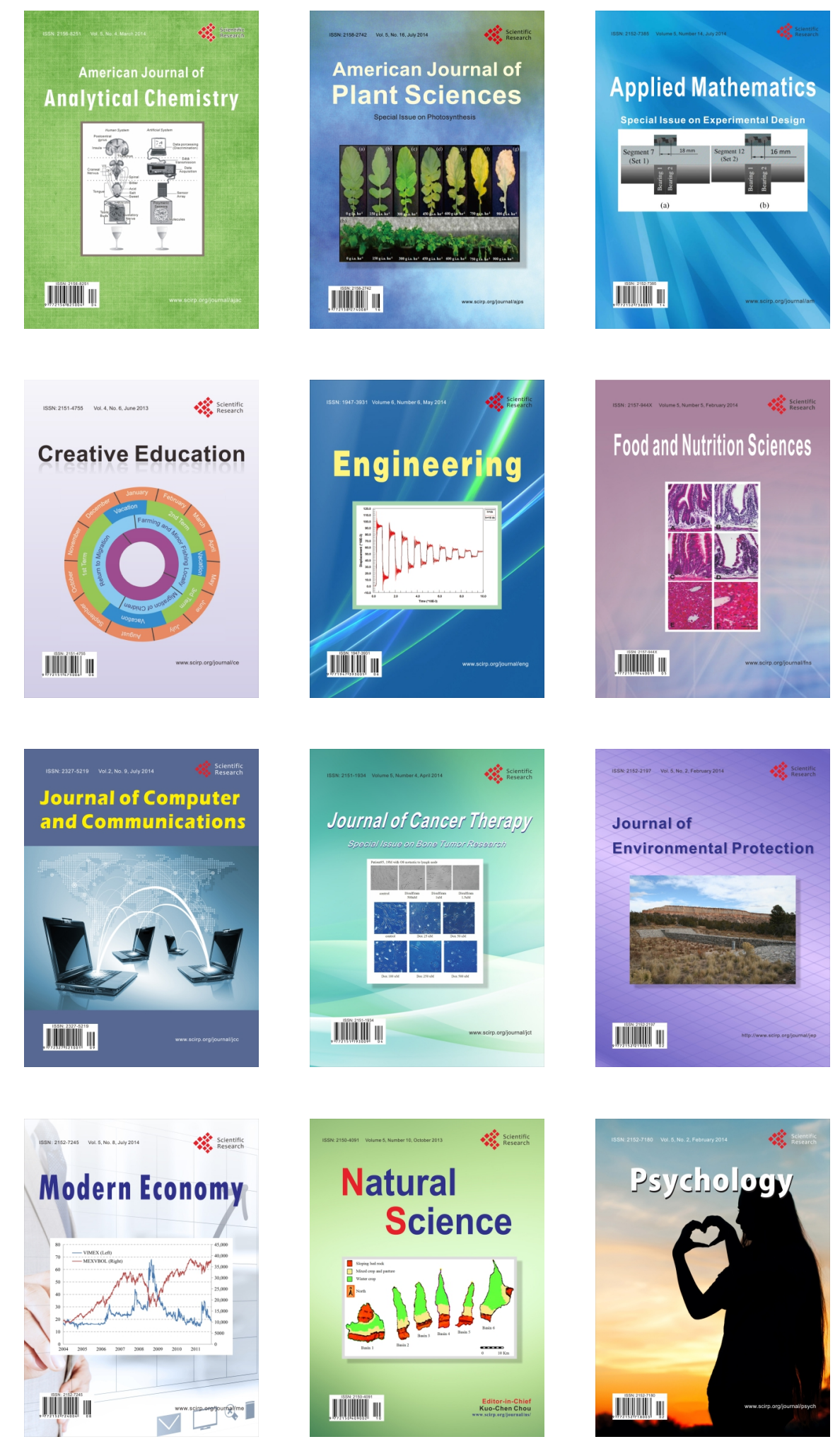